\title{
Environment-aware Proximity Detection with Capacitive Sensors for Human-Robot-Interaction
}

\author{
Alwin Hoffmann, Alexander Poeppel, Andreas Schierl, and Wolfgang Reif
}

\begin{abstract}
Recently, the need for safe human-robotinteraction has become increasingly important, and with it the requirement to reliably detect persons in the workspace of a robot. Capacitive sensors mounted to the robot structure can be used to measure the presence of conductive objects and, hence, allow the detection of persons. However, various objects in the workspace can influence capacitive sensor measurements. Thus, we propose to record an environment model containing the expected sensor values for relevant robot poses. Using this model, distance estimation and real-time reaction can be performed even in the presence of additional conductive objects in the workspace. A demonstration of our approach was shown at the Hannover Messe 2015.
\end{abstract}

\section{INTRODUCTION}

Currently, the interest in safe human-robot-interaction is increasing. The reasons for this include attempts to automate tasks that need cooperation as they cannot be executed by a robot alone, or to reduce cycle times by introducing a common workspace. The prospect of demographic changes, specifically the increased ageing of the population, is another cause, which leads to the expectation of robots being capable of assisting senior workers by relieving them of inconvenient and strenuous work postures. In order to implement safe human-robot-interaction, collisions have to be avoided or at least reduced to a non-hazardous level. To detect humans and other objects within the workspace, various methods have been proposed or employed:

Especially for light-weight robots, methods utilizing the measurement of motor currents or torques have been implemented. A prominent example is the DLR lightweight manipulator arm (cf. [1], [2]) which is currently commercially available from KUKA in form of the LBR iiwa. Other research utilizes tactile sensors mounted on robot links (cf. [3], [4]). These approaches have the common disadvantage that collisions can only be detected after they occurred, but cannot be anticipated and avoided. Cameras (either merely RGB, or such including depth information) have also been used in this field (cf. [5], [6]). The main drawback, however, is the problem of shadowing in the environment, either through objects or the structure of the robot itself. To compensate for this, cameras for detection of humans in the workspace have been combined with safe physical collision detection mechanisms. For example, in [7]

The authors are with the Institute for Software and Systems Engineering, University of Augsburg, D-86135 Augsburg, Germany. E-mail of corresponding author: hoffmann@informatik. uni-augsburg. de

This work partly presents results of the research project SafeAssistance which is funded by the German Federal Ministry of Education and Research (BMBF). The project was carried out together with MRK-Systeme GmbH and was kindly supported by VDI/VDE-IT GmbH. a visual safety system, which detects the presence of humans, and a passive control approach using joint impedance are combined to guarantee safe reaction even in worst-case scenarios. In [8], a collision avoidance system based on depth information is used in combination with physical collision detection based on residual signals of joint positions. Another possible system for detecting collisions before they occur is the use of ultrasonic sensors mounted on the robot structure, however the field of detection, as well as the highest possible update rate are severely limited. Moreover, the problem of interference when using multiple sensors of this type simultaneously is highly pronounced, as sound waves are reflected by any solid surface (cf. [9]).

Hence, capacitive sensors have been used to secure robots since the 1990s (cf. [10], [11]), with various improvements over time. A variant combining tactile sensors for safety stops with capacitive sensors for remote detection (cf. [12], [13]) is already commercially available (cf. KR 5 SI [14]), however with some issues concerning influences of the environment. Additionally, current work [15] emphasizes the need for quick measurement and reaction in order to safely halt the robot, which leads to novel measurement principles with a higher signal to noise ratio [16]. Similarly, in [17] a modular electronics platform for constructing a matrix of capacitive-tactile proximity sensors has been presented, which can be used for combined proximity- and touch-based interaction of humans with robots. In summary, capacitive sensors - especially when mounted directly on a robot arm - are highly relevant in the field of safe human-robotinteraction [18].

However, the main issue when using capacitive sensors for human-robot-interaction is that not only humans, but generally any conductive material influences the sensor value. Without any knowledge of the environment, it is impossible to distinguish between static obstacles (where no collisions are to be expected if the robot is programmed properly) and dynamic, typically human obstacles (with which a collision must be avoided). This is especially important when capacitive sensors are employed for proximity detection in ranges greater than $5 \mathrm{~cm}$. As these issues have not been addressed before, the main contribution of this paper is to introduce an environment model containing information about static objects in the workspace and thus allowing to reliably distinguish between these objects and additional, dynamic obstacles.

This paper is structured as follows: First, the idea of distance estimation using capacitive sensors is explained (see Sect. II). Subsequently in Sect. III, our solution to miti- 


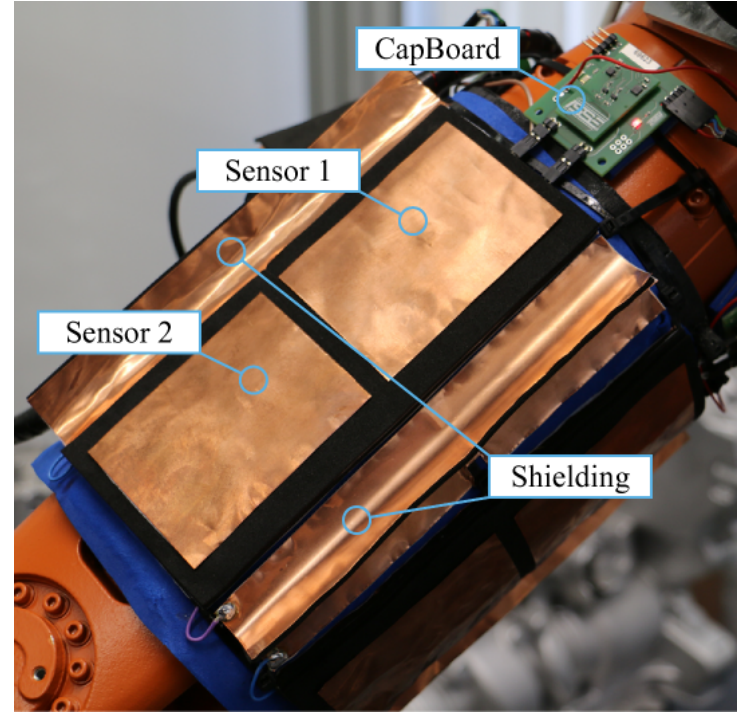

Fig. 1. Capacitive sensors are mounted at the structure of a KUKA KR 6 AGILUS. Each measurement circuit is connected to two electrodes. These are surrounded by an actively modulated shielding plane. The possible detection range of this configuration extends up to a distance of approximately $35 \mathrm{~cm}$.

gate influences of the environment through a pre-recorded environment model is introduced, including a procedural description of how to record the required data with sufficient quality. Sect. IV presents the implementation of the proposed capacitive sensor system. This includes the electronics for the sensing device as well as the software for the environmentaware proximity detection. A short evaluation is given in Sect. V including possible reaction strategies for avoiding collisions based on the gathered data. The approach is demonstrated in an inspection task application for the automotive industry which was shown at the Hannover Messe 2015. Sect. VI concludes the paper with a summary of the main findings gathered in our work.

\section{DISTANCE ESTIMATION}

To detect persons in the workspace of the robot and to avoid collisions, capacitive sensors are mounted on the robot structure (cf. Fig. 1). These sensors consist of two layers of copper foil, serving as excitation and measurement electrodes respectively, in conjunction with a custom measurement circuit (see Sect. IV), and are used to determine the capacitance between the measurement electrode and the ground potential. The measured capacitance $c$ is expressed in the corresponding physical unit of Farad (F).

When plotting the measured capacity against the distance of a present object (cf. Fig. 2), two aspects become obvious. First, keeping the environment constant (i. e. nothing except the hand moves, in particular the sensor keeps the same position), there is a non-linear relation between the measured capacitance and the distance between the hand and the sensor. And second, the addition of a static obstacle (while keeping the remaining environment constant) increases the measured value by a fixed offset, which does not depend on the distance to the human hand.

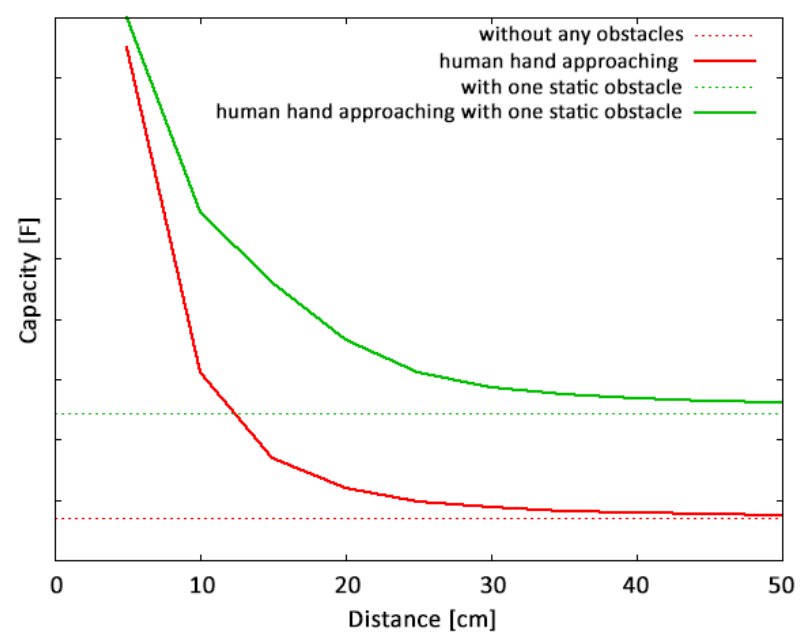

Fig. 2. Measured sensor signals for different scenarios approaching the capacitive sensor with a human hand. Static obstacles add a constant offset to the sensor signal.

Thus, it is possible to define a distance function $\mathrm{s}: \mathbb{R} \mapsto \mathbb{R}$ that maps the difference between a sensor's current value and its base capacitance, termed $\Delta c$, to a hand distance $s_{\text {hand }}$ :

$$
\mathrm{s}: \Delta c \rightarrow s_{\text {hand }}
$$

It is preferable to choose the hand as reference for the capacitive values, as it constitutes the smallest body part of human beings which is of interest for the detection in this context. Any other part which could enter the workspace of the robot is larger and will therefore have a greater influence on the measured capacitance value. In consequence, the distance function is not an exact calculation, but rather an estimation of the minimal distance to an object.

For any given sensor geometry, this distance function can be determined using measurements as the ones shown in Fig. 2. Effectively, this is accomplished by approaching the sensor with a hand and recording the measured capacitance values in correlation with independently determined distance measurements. Afterwards, a function approximation can be performed, to find the specific function inherent to the desired sensor geometry. In our evaluation, we performed a nonlinear least squares fitting. Out of all possible results, the function with the lowest error in the desired value range was chosen. It should be noted that the capacitive values must be limited to the range corresponding this desired distance range to prevent undesired results.

One major concern, when using capacitive sensors for distance estimations in safety relevant applications, is the sensor drift caused by temperature changes in the surrounding environment. While a high temperature value results in a higher than usual capacitance value, and thus eventually causes a halt of the robot, a low temperature which corresponds to a lower capacitance causes a negative offset and leads to a delayed, or in the worst case no reaction. Though both effects are undesirable, the latter poses a significant safety risk for use in human-robot-interaction. To eliminate this 
problem, we devised a system to counteract the influence of temperature fluctuations on capacitance values. By encasing an electrode pair, identical to those used at the robot, in a Faraday cage, a reference value for all other sensors can be established. Then at runtime, the difference between its initial and current capacity value must be continuously calculated to subsequently offset each of the remaining sensor values.

\section{ENVIRONMENT MODEL}

Due to the results shown in Fig. 2, it is possible to compensate for static influences created by the components of the robot cell, i. e. the peripherals, work-pieces and further common obstacles inside the robot's workspace. As the robot is moving and with it the capacitive sensors, these influences vary for every position. Hence, an environment model containing the base values of the capacitive sensors at each of these points can be created initially and used during runtime to improve the proximity estimation. This environment model must capture the relevant part of the robot's workspace, i.e. at least all paths traversed by the robot program must be represented within it.

The environment model is a function e which retrieves a vector $\vec{c} \in \mathbb{R}^{m}$ of $m$ sensor values for a given vector $\vec{q} \in \mathbb{R}^{n}$ of $n$ joint angles. The position of the robot is specified using joint angles rather than a position in Cartesian space, since the grounded robot structure itself also influences the measurements. Thus, a three-dimensional point in space, which is reachable through multiple different axis configurations, must possess distinct base values for each possible robot pose. The function is defined as follows:

$$
\mathrm{e}: \vec{q} \rightarrow \vec{c}
$$

This function can be implemented by collecting sensor values for the relevant fragment the workspace into an appropriate data structure for later comparison with current values.

However, since recording capacitive sensor data for every possible joint position of the robot would be extremely time consuming, it is more efficient to periodically sample the sensor values during workspace exploration. To this end, the robot initially traverses its static environment (i.e. without any persons present) with its operational velocity, while the control system records the values of the attached capacitive sensors at maximum sampling rate and creates a set $M \subset$ $\mathbb{R}^{n} \times \mathbb{R}^{m}$ in which every point is a combination of a vector of $n$ joint angles (where $n=6$ for a standard industrial robot) and $m$ sensor values.

As this environment model is the only source of information regarding the robot's environment at runtime, the recorded sensor values must be highly reliable. However, capacitive sensor measurements contain statistical noise which is assumed to be drawn from a zero mean normal distribution with unknown variance $\sigma^{2}$. Hence, we propose to gather a large amount of sensor data over multiple exploration cycles and perform statistical analyses in order to improve the quality of stored (expected) sensor values and, by doing so, reducing sensor noise from the environment model.
After multiple exploration cycles, it is necessary to process the resulting data using a clustering algorithm which groups the sensor values by their corresponding joint angles. To effectively limit the distance between two cluster points in Cartesian space, a weighted comparison was performed to determine a point's membership to a given cluster. We define a vector $\vec{r} \in \mathbb{R}^{n}$ over $n$ joint values, which represents the allowed radius around the base point $\vec{q}_{b}$ of a cluster. Within this radius, a data point $\vec{q}$ is considered a part of the cluster. Thus, a cluster $C$ around a base point $\vec{q}_{b}$ is defined as follows:

$$
C:=\left\{\vec{q} \in \mathrm{q}(M) \mid \forall i \in \mathbb{N}, i<n: q_{b}^{i}-r^{i}<q^{i}<q_{b}^{i}+r^{i}\right\}
$$

where $\mathrm{q}: \mathbb{R}^{n} \times \mathbb{R}^{m} \mapsto \mathbb{R}^{n}$ is a function selecting the joint angles from $M$. Using this clustering algorithm, the environment model is reduced to the most relevant (base) points. These points are gradually selected during clustering.

Afterwards, an estimate for the expected sensor values at a given position can be determined by calculating the arithmetic sample mean for each sensor from all data points contained in a cluster. To further improve the resilience against noise-impacted sensor values, we propose to calculate a prediction interval around the sample mean for each sensor value. We assume that sensor measurements are given by a normal distribution $N\left(\mu, \sigma^{2}\right)$ with both $\mu$ and $\sigma^{2}$ unknown. While $\mu$ represents the true sensor value, $\sigma^{2}$ defines the measurement noise. As both $\mu$ and $\sigma^{2}$ are unknown, the prediction interval should be calculated using the sample variance and the Student's t-distribution for a required confidence (e.g. 95\%). It is sufficient to merely store the maximum value of the resulting predication interval as an upper estimate for each sensor value as the system is intended to react to unknown objects entering the workspace, and thus should not react prematurely to higher sensor readings within an acceptable noise band.

In consequence, a mapping of cluster base points and upper estimates for the expected sensor value is stored into the final environment model. Later, during runtime, a nearest-neighbor search is performed to find the point closest to a current joint position $\vec{q}$ and the corresponding vector of expected sensor values $\vec{c}$ is returned (cf. Sect. IVB). In order to augment performance of data storage and organization, space-partitioning methods (e. g. k-d trees [19]) can be employed. Using the distance estimation (cf. Sect. II) in conjunction with the world model, it is possible to merely determine the distance of dynamic obstacles, thus eliminating the necessity of reacting to every influence registered by the capacitive sensors.

\section{IMPLEMENTATION}

We implemented the environment model and the distance estimation using the SoftRobot architecture [20]. There robotics software is developed against an application programming interface (API) - the Java-based Robotics API [21] - whereas robot operations are executed with hard realtime guarantees within the underlying Robot Control Core $(R C C)$ [22]. Thus, the RCC is responsible for directly con- 


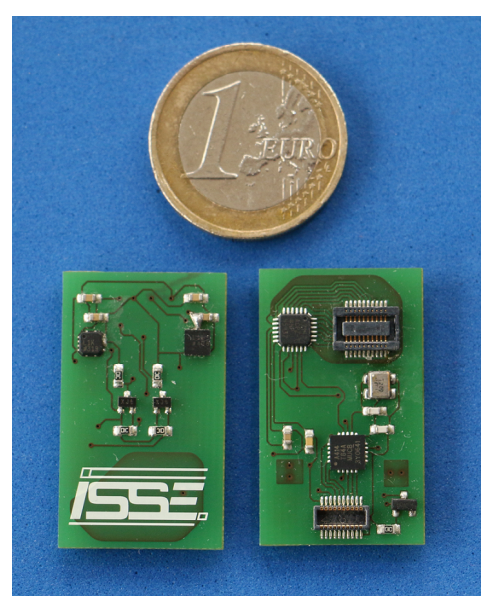

Fig. 3. CapBoard: a modular custom circuit using the AD7147 capacitive sensor IC from Analog Devices which provides 12 sampling stages and 13 hardware inputs for capacitive sensors. Due to its small size, it can be mounted at the robot arm close to the capacitive sensors.

trolling hardware devices and is implemented on a real-time operating system (i.e. Linux with Xenomai extensions).

Hence, the RCC takes care of all real-time critical parts of the robotics systems and provides a flexible, generic interface: the Realtime Primtives Interface (RPI) [23]. RPI is a dataflow based language, consisting of basic calculation modules, which can be combined to form complex commands (e.g. the distance estimation). These compositions of calculation modules are executed periodically with a frequency of up to $1 \mathrm{kHz}$ with real-time guarantees. Besides these basic calculation modules, there are also real-time device modules for sending data to or retrieving data from sensors and actuators (e.g. the capacitive sensing devices and the robot, respectively).

\section{A. Sensing Device}

To achieve the desired accuracy for the capacitive sensors in combination with an optimized sensor topology on the robot arm, a custom circuit was designed (CapBoard, see Fig. 3), using the AD7147 capacitive sensor chip (cf. [24]) from Analog Devices. Due to the small form factor of the designed circuit board, it becomes possible to attach the measurement device in direct proximity of the sensor electrodes on the robot, and thus eliminating the need for long sensor cables running along the robot arm, which introduce capacitance shifts under mechanical stress. This integrated circuit (IC) provides 12 sampling stages and 13 hardware inputs for capacitive sensors, whereby the mapping between these two is user configurable. In our evaluation, the chip was configured to measure 2 inputs with 6 stages each every $9.2 \mathrm{~ms}$. Additionally, a ATTiny $84 \mathrm{~A}$ microcontroller from Atmel (cf. [25]) was included for aggregation and processing of the data received from the sensor chip. The external communication with the RCC is implemented using a bus interface, which allows for seamless integration of any required number of capacitive sensors into the robot application. Another microcontroller is required to serve as bus master and intermediary between the low-level bus system and more advanced communication systems found in modern computers. In our evaluation, this task was handled by an Arduino MEGA 2560 equipped with an Ethernet shield, which is configured by the control system with the bus addresses of the attached CapBoards and is subsequently able to relay the gathered sensor data from all of these to the computer which executes the robot application.

Since the high frequency modulations created by the capacitive sensor ICs during measurement cycles interfere with each other when in close proximity to one another, a method of guaranteeing exclusive access to adjoining electrodes within a group of sensors had to be devised. Due to the fact that any type of grounded shielding between sensors impedes the detection range they can provide, as the capacitance between an electrode and the ground potential is the parameter to be measured, a different method had to be developed, in order to maintain the desired sensor range and stability. The integration of a time slot mechanism into the firmware of the CapBoard provided the best compromise over all requirements. Hereby, every CapBoard receives a designated time slot, in which it enables the AD7147, gathers the sensor data, and disables it again. This process takes between 15 and $20 \mathrm{~ms}$, and consequently a slot duration of $25 \mathrm{~ms}$ was chosen. Employing this method makes it possible to guarantee exclusive access to the sensors for each IC, while maintaining the previously attained detection range of a single sensor. The downside to this approach is the reduction of the measurement frequency per sensor in each group to

$$
f=\frac{1}{0.025 \mathrm{~s} * \text { number of sensors }}, \text { with }[f]=\mathrm{Hz}
$$

However, using between 2 and 4 sensors within one time slot group, the measurement frequency remains sufficient to quickly and accurately detect humans entering the workspace of the robot.

\section{B. Environment-Aware Proximity Detection}

By combining all results previously described in this paper, the system is able to reliably detect the presence of a person in the workspace of a robot, independent of any native obstacles. As the robot moves along the programmed paths through the workspace, the current and expected values are continuously compared with each other and subsequently a distance estimation is performed (cf. Fig. 4). To this end, a vector of expected capacitance values is retrieved from the world model in each execution cycle. This is accomplished by implementing an approximate nearest-neighbor search on the stored environment model. In this case, we chose the Fast Library for Approximate Nearest Neighbors (FLANN, cf. [26]) which was directly integrated into the $R C C$ for fast and efficient data queries. The expected value is subtracted from the currently measured capacitance $\vec{c}$ to obtain the quantitative influence $\Delta c$ of foreign objects entering the workspace. Hence, we are able to perform the previously mentioned distance estimation on the results, and finally 


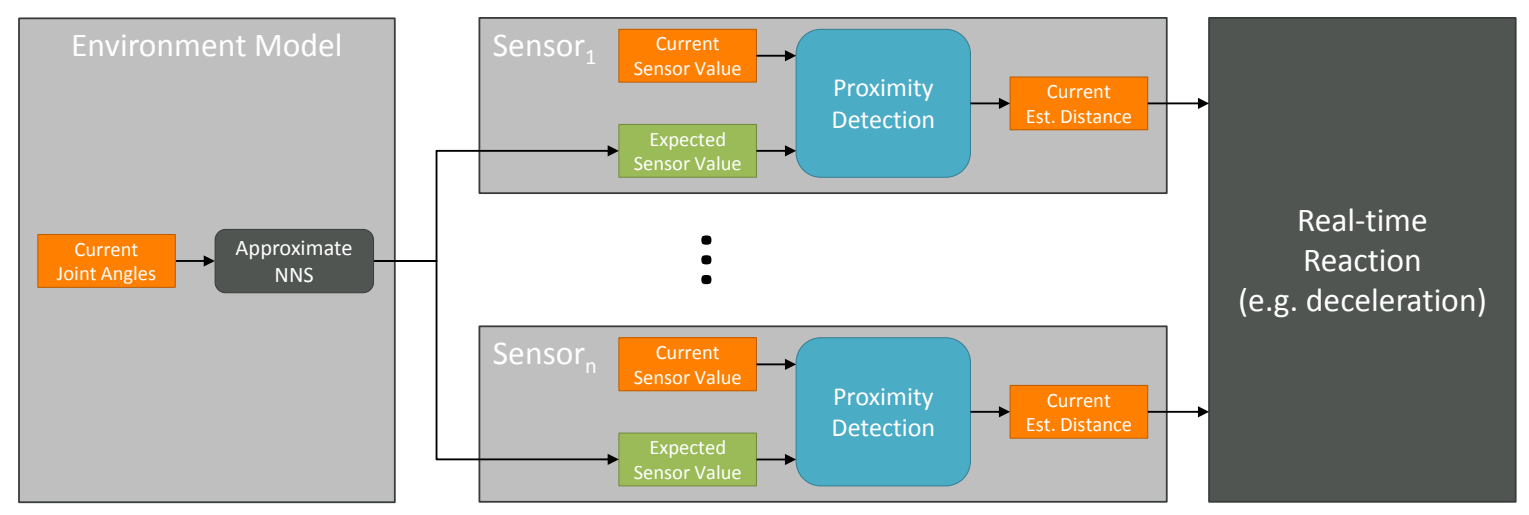

Fig. 4. In each cycle the expected nominal sensor value is retrieved using an approximate nearest-neighbor search (NNS) on the environment model and compared to the current sensor signal. Based on this comparison the current distance to an assumed person in the robot's workspace is estimated. Subsequently, an appropriate reaction of the robot is calculated (e.g. decelerating).

receive a vector $S \in \mathbb{R}^{m}$ containing the estimated distance to a dynamic obstacle for each sensor with $m$ as formerly mentioned being the number of sensors attached to the robot. Each element of this vector (denoted by the index $i$ ) is therefore calculated by the function:

$$
S_{i}=\mathrm{s}\left(\max \left(0, c_{i}-\mathrm{e}(\vec{q})_{i}\right)\right)
$$

with a limitation to positive values as negative values can be considered due to measurement noise.

An additional noteworthy aspect is that all capacitive values, the current ones, as well as those contained in the world model, are at all times compensated with respect to the influence of temperature, thus creating long term comparable data and eliminating the need for repeated recording of the environment model. In conjunction, all these procedures facilitate acceptable distance estimations in spite of environments with a multitude of influences and disturbances. Hence, the proposed system enables the development of robotics applications with arbitrary reaction strategies based solely on distance information, while under the assurance that the normal operation of the robot will not be impeded by static obstacles native to the workspace.

\section{EVALUATION}

The presented approach was successfully evaluated using a UR 5 from Universal Robot as well as a KR 6 AGILUS from KUKA. For the latter robot, a demonstration cell was realized for the Hannover Messe 2015 (cf. Fig. 5). The task is to inspect car engines with a camera mounted at the robot's flange. The static obstacles influencing the capacitive sensors are the four engine blocks, the flat panel displays and the robot structure itself. In total, there are four capacitive sensors mounted on the robot. Three sensors are mounted at the arm sensing to the right, to the left and to the bottom. The fourth capacitive sensor is mounted around the camera at the flange. The video attachment ${ }^{1}$ shows a series of experiments using this setting.

\footnotetext{
${ }^{1}$ The video attachment is available in high definition at: http:// video.isse.de/safeassistance/iros2016
}

While the proposed sensor system opens up any number of possible reaction strategies, we chose a simple one to objectively evaluate the overall performance and reactivity of the developed system. It shows that the robot gradually decreases velocity when approaching a dynamic obstacle, but is generally unaffected by the entirety of additional influences throughout the workspace. As an additional feature, we added the ability to react to obstacles based on the relationship between the motion direction of sensors and the dynamic object entering the workspace. Using the Robotics $A P I$, we have been able to continuously calculate for each sensor whether it is moving in the direction of its detection field, merely by providing the static sensor positions on the manipulator. We can then compare the resulting motion direction, which is defined orthogonally on the sensor plane, with the difference between current and previously measured distances, to determine whether the sensor is moving away from or towards the obstacle in question.

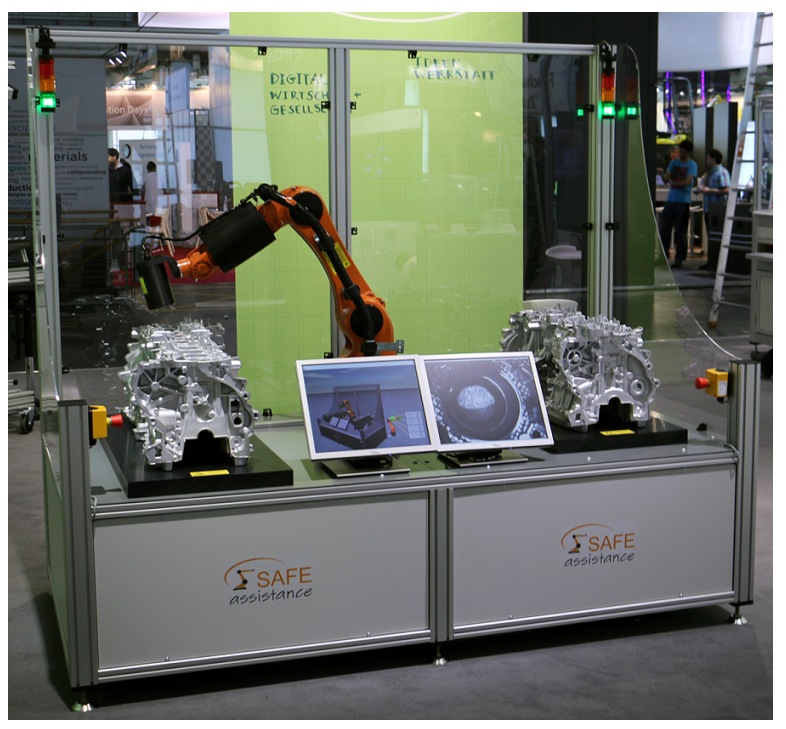

Fig. 5. Demonstration cell with a KR 6 AGILUS at the Hannover Messe in 2015 showing an inspection task for automotive industries. There are multiple capacitive sensors mounted on the arm and on the tool. 
Performing this calculation for each sensor enables the implementation of more complex reaction strategies. For example, we used this information to modify the braking ramp of the robot and provide a more natural interaction experience with the robot. With the addition of direction information for each sensor, the application is also able to determine how the robot as a whole is moving in relation to an obstacle and can react accordingly. In our example, we implemented a deferred braking ramp for cases wherein no sensor reports that it is moving towards an obstacle, even if the object itself is moving in the same direction. A halt of the robot is then precipitated by the distance to an object falling below a predefined safety threshold. Through this method, a large amount of perceivedly unnecessary halts of the robot could be eliminated, thus creating a more desirable user experience.

\section{CONCLUSION}

The main contribution of this paper is a novel approach to overcome the typical drawbacks of capacitive sensors for reliably detecting objects in a structured (industrial) environment. By using a previously recorded environment model, the current sensor values can be compared to the expected values, and consequently the distance to a dynamic object (e. g. a human hand or arm) be estimated. Through statistical computation, the sensor noise can largely be eliminated from the gathered data, thus providing a more reliable map of the static obstacles in the robot's environment. On this basis, we were able to implement exemplary reaction strategies to evaluate the overall system performance. Our demonstration cell shows promising results in terms of reactivity and usability for the field of human-robot-interaction.

Our future work will continue to improve the sensing device, e.g. by surveying further capacitive measurement principles and evaluating their performance with respect to possible detection range and noise immunity. Another avenue of research will focus on improving the resolution of the proximity detection, possibly by significantly increasing the number of sensors attached to the robot, in order to be able to implement more complex reaction strategies like an impedance mode. Furthermore, we plan to explore the possibility of creating a universal environment model through heuristic algorithms and machine learning, which could be recorded independently of concrete robot applications. In combination with further sensors, such a model could be capable of working in dynamic, less structured environments.

\section{ACKNOWLEDGMENT}

The authors would like to thank Andreas Angerer, Miroslav Macho, Ludwig Nägele, Michael Vistein, and Constantin Wanninger for their help in realizing the demonstration cell. Moreover, the authors would also like to thank Peter Heiligensetzer and MRK-Systeme GmbH for their support.

\section{REFERENCES}

[1] A. Albu-Schäffer, C. Ott, and G. Hirzinger, "A unified passivitybased control framework for position, torque and impedance control of flexible joint robots," Int. J. Rob. Research, vol. 26, no. 1, pp. 23-39, 2007.
[2] A. De Luca, A. Albu-Schaffer, S. Haddadin, and G. Hirzinger, "Collision detection and safe reaction with the DLR-III lightweight manipulator arm," in Proc. 2006 IEEE/RSJ Intl. Conf. on Intell. Robots and Systems, Beijing, China, 2006, pp. 1623-1630.

[3] P. Mittendorfer and G. Cheng, "Humanoid multimodal tactile-sensing modules," IEEE Trans. on Robotics, vol. 27, no. 3, pp. 401-410, 2011.

[4] E. Cheung and V. Lumelsky, "A sensitive skin system for motion control of robot arm manipulators," Robotics and Autonomous Systems, vol. 10, no. 1, pp. 9-32, 1992.

[5] T. Gecks and D. Henrich, "Human-robot cooperation: Safe pickand-place operations," 14th IEEE Intl. Works. on Robot and Human Interactive Comm., Nashville, USA, 2005.

[6] L. Wang, B. Schmidt, and A. Y. Nee, "Vision-guided active collision avoidance for human-robot collaborations," Manufacturing Letters, vol. 1, no. 1, pp. 5-8, 2013.

[7] R. Schiavi, A. Bicchi, and F. Flacco, "Integration of active and passive compliance control for safe human-robot coexistence," in Proc. 2009 IEEE Intl. Conf. on Robot. \& Autom., Kobe, Japan, 2009, pp. 259-264.

[8] A. De Luca and F. Flacco, "Integrated control for phri: Collision avoidance, detection, reaction and collaboration," in Proc. 4th IEEE RAS EMBS Intl. Conf on Biomedical Robotics and Biomechatronics, Rome, Italy, 2012, pp. 288-295.

[9] B. Ostermann, M. Huelke, and A. Kahl, "Freed from fences: safeguarding industrial robots with ultrasound," Safety Science Monitor, vol. 17 , no. $1,2013$.

[10] J. Novak and J. Feddema, "A capacitance-based proximity sensor for whole arm obstacle avoidance," in Proc. 1992 IEEE Intl. Conf. on Robot. \& Autom., Nice, France, 1992, pp. 1307-1314.

[11] J. T. Feddema and J. L. Novak, "Whole arm obstacle avoidance for teleoperated robots," in Proc. 1994 IEEE Intl. Conf. on Robot. \& Autom., San Diego, CA, USA, vol. 4, 1994, pp. 3303-3309.

[12] P. Heiligensetzer, "Sichere Mensch-Roboter-Kooperation durch Fusion haptischer und kapazitiver Sensorik," Diss., Univ. Karlsruhe, 2003.

[13] R. Koeppe, D. Engelhardt, A. Hagenauer, P. Heiligensetzer, B. Kneifel, A. Knipfer, and K. Stoddard, "Robot-robot and human-robot cooperation in commercial robotics applications," in 11th Intl. Symposium on Robotics Research. Springer, 2005, pp. 202-216.

[14] KR 5 SI (SafeInteraction), MRK-Systeme GmbH, http://www.mrksysteme.de/downloads/Prospekt_KR_5_SI_en.pdf.

[15] T. Schlegl, T. Kröger, A. Gaschler, O. Khatib, and H. Zangl, "Virtual whiskers - highly responsive robot collision avoidance." in Proc. 2013 IEEE/RSJ Intl. Conf. on Intell. Robots and Systems, Tokyo, Japan, 2013, pp. 5373-5379.

[16] T. Schlegl and H. Zangl, "Capacitive sensing for safety applications," in Technologies for Smart Sensors and Sensor Fusion, K. Yallup and K. Iniewski, Eds. CRC Press, 2014, ch. 15, pp. 259-282.

[17] S. Escaida Navarro, M. Marufo, Y. Ding, S. Puls, D. Göger, B. Hein, and H. Wörn, "Methods for safe human-robot-interaction using capacitive tactile proximity sensors," in Proc. 2013 IEEE/RSJ Intl. Conf. on Intell. Robots and Systems, Tokyo, Japan, 2013, pp. 1149-1154.

[18] S. Escaida Navarro, B. Hein, and H. Wörn, "Capacitive tactile proximity sensing: From signal processing to applications in manipulation and safe human-robot interaction," Soft Robotics, pp. 54-65, 2015.

[19] J. L. Bentley, "Multidimensional binary search trees used for associative searching," Commun. ACM, vol. 18, no. 9, pp. 509-517, 1975.

[20] A. Hoffmann, A. Angerer, F. Ortmeier, M. Vistein, and W. Reif, "Hiding real-time: A new approach for the software development of industrial robots," in Proc. 2009 IEEE/RSJ Intl. Conf. on Intell. Robots and Systems, St. Louis, MO, USA, 2009, pp. 2108-2113.

[21] A. Angerer, A. Hoffmann, A. Schierl, M. Vistein, and W. Reif, "Robotics API: Object-oriented software development for industrial robots," J. of Software Engineering for Robotics, vol. 4, no. 1, pp. $1-22,2013$.

[22] M. Vistein, A. Angerer, A. Hoffmann, A. Schierl, and W. Reif, "Flexible and continuous execution of real-time critical robotic tasks," Intl. J. Mechatronics \& Autom., vol. 4, no. 1, 2014.

[23] _ , "Interfacing industrial robots using realtime primitives," in Proc. IEEE Intl. Conf. on Autom. and Logistics, Hong Kong, 2010.

[24] AD7147: CapTouch Programmable Controller for Single-Electrode Capacitance Sensors, Analog Devices, Norwood, MA, USA, 2015.

[25] ATtiny24A/44A/84A, Atmel Corporation, San Jose, CA, USA, 2012.

[26] M. Muja and D. G. Lowe, "Fast approximate nearest neighbors with automatic algorithm configuration," in Intl. Conf. Computer Vision Theory and Application. INSTICC Press, 2009, pp. 331-340. 